\title{
SIMULATION OF PHOTOGRAMMETRY-BASED 3D DATA ACQUISITION
}

\author{
Gajic, D. B. ; Mihic, S. ${ }^{* *}$; Dragan, D. ; Petrovic, V. ${ }^{*} \&$ Anisic, Z. \\ * University of Novi Sad, Faculty of Technical Sciences, Trg Dositeja Obradovica 6, Novi Sad, Serbia \\ ${ }^{* *}$ Doob Innovation Studio, Bulevar oslobodjenja 127/V/40, Novi Sad, Serbia \\ E-Mail: dusan.gajic@uns.ac.rs, s.mihic@doobinnovation.com,dinud@uns.ac.rs,pveljko@uns.ac.rs, \\ anisic@uns.ac.rs
}

\begin{abstract}
The paper presents a simulation approach to photogrammetry-based three-dimensional (3D) data acquisition. Photogrammetry requires capturing of series of overlapping photos with certain properties from which 3D reconstruction is later obtained. Scanning a building or a human or jewellery requires different numbers of cameras, setup parameters, spatial orientations, etc. Without precise information on how to effectively take photos, obtaining them can be tedious work without any guarantees that it will provide sufficient $3 \mathrm{D}$ reconstruction quality. The proposed simulation approach aims to ease the aforementioned burdens and contributes by improving the process of photogrammetry-based 3D data acquisition. The presented simulator is tested in the context of the development of a 3D scanning system for human body scanning and avatar creation. The experiments confirm that the proposed method leads to an improved quality of 3D object reconstruction in comparison to previous practice in the field of 3D human scanning. Further, it lowers the cost and shortens the time required for the industrial process of construction of 3D scanning systems, thus confirming the value and validity of the presented approach.

(Received in September 2018, accepted in January 2019. This paper was with the authors 2 weeks for 1 revision.)
\end{abstract}

Key Words: Simulation Software, 3D Data Acquisition, Photogrammetry, Human Body Scanning, Avatars

\section{INTRODUCTION}

Due to the introduction of affordable three-dimensional (3D) scanners to mass market and development of effective and cheap 3D scanning techniques, the number of 3D digitization projects has been steadily and rapidly increasing in recent years [1-3]. It should be noted that digitalization and digitization are distinct concepts. Digitalization describes the enhancement of existing business programs with digital technologies [4]. Digitization, however, is changing from analogue to digital, in this paper's case, from physical representation to digital 3D object. Applications of 3D digitization significantly vary, from preserving cultural heritage objects or topographic mapping of entire cities, to making avatars of human beings and improving industrial production processes [2, 3, 5-7]. A wide variety of 3D scanning techniques are being employed for 3D digitization, an overview of which can be found in the extensive literature [2, 8-11] on the subject.

Digital photogrammetry, as an affordable 3D data acquisition/scanning technique that allows detailed 3D reconstruction of scanned objects, has become very popular and it is broadly used in a variety of fields $[2,10]$. It represents a technique for acquiring dense 3D geometric data of physical objects from stereoscopic photograph overlap. Photogrammetry requires a series of overlapping photographs taken of the physical object, usually with common digital (still) cameras, from which 3D reconstruction is obtained using 3D photogrammetric software. Many factors influence the success of 3D reconstruction of scanned objects, such as positions of cameras, their orientations, amount of overlap in photos and their sharpness, lighting conditions, and coverage of the scanned physical object. 
Different applications of photogrammetry differ in parameters necessary for optimal 3D reconstruction quality. Parameters such as the number of photographs required, their distance from the target and orientation may differ significantly. Some guidelines regarding these parameters exist in the literature [1], but, to the best of our knowledge, no definitive and universal set of rules exists. Without such precise information, taking sufficient number of photographs can be tedious work without any guarantee that it will provide 3D reconstruction of desired quality. This presents a major problem for the efficient design and construction of 3D scanning systems which should reliably produce adequate 3D reconstructions.

The main contribution of the simulation approach presented in this paper is in narrowing the aforementioned information gap and thus allowing more efficient photogrammetry-based $3 \mathrm{D}$ data acquisition. We apply the proposed simulator to the process of optimization of 3D scanning chamber development and production. These chambers are built for human body scanning and avatar creation. We show that the proposed simulator lowers the costs and shortens the time required for 3D scanner design and construction in comparison to the current industrial best practices. We also demonstrate that 3D human scanners, built using our proposed simulator, are characterized by improved quality of achieved 3D object reconstruction when compared to 3D scanners previously in use. To the best of our knowledge, this work is the first proposing a simulator of 3D data acquisition in a close-range photogrammetry use-case, and which goes on to use this simulator for 3D scanning system design and construction optimization, as well as improving scanner performance.

The remainder of the paper is organized as follows. In Section 2 we give an overview of the work related to the presented research. Section 3 describes the problem statement and the applied methodology. Details of the proposed simulator are elaborated upon in Section 4. Experimental settings and results are discussed in Section 5. The closing section offers main conclusions from the presented research and gives directions for the future research.

\section{RELATED WORK}

A detailed description of the field of close-range photogrammetry and 3D imaging can be found in [10]. In [2], Hartley and Zisserman give a general overview of the multiple view geometry methods in computer vision, including the Structure-from-Motion (SfM) algorithms which we use in our simulator. The application of some other techniques from computer vision for monitoring of industrial processes can be found in [12]. A more recent look at both the incremental and global SfM techniques is given in [8]. Once the 3D reconstruction has been created using the $S f M$ algorithms, the accuracy of the obtained 3D model needs to be measured. Zhao and Loy present an approach to determining the accuracy and resolution of 3D models acquired originally via 3D digitization [1].

Simulation based approaches, similar to the one proposed in this paper, have proved to be successful in various application domains. Piatti and Lerma present a virtual world for photogrammetric image-based simulation [3]. The presented simulator mainly targets image acquisition by unmanned aerial vehicles (UAVs). It is used to create $3 \mathrm{D}$ virtual worlds in order to improve the understanding of UAV mission planning. They also generate synthetic image sequences with suitable overlaps and thus try to optimize image data acquisition before undertaking expensive photogrammetric surveys in the field [3]. The simulator presented in this paper aims to solve similar problems which are discussed in [3], but in the context of 3D scanning of humans in order to create their avatars using close-range photogrammetry techniques $[13,14]$.

Simulation approaches, similar to the one presented in this paper, have also proven useful in improving industrial production processes, e.g., modelling of the metal casting processes [15] and simulation and analysis of ball-end milling of panel moulds [16], as well as for solving problems such as improvement of workplace design which considers ergonomics and 
productivity [12]. An approach to performance analysis based on simulation in the narrow context of mass storage systems is described in [17]. The application of simulation software as a tool for the analysis and performance improvement, which is basically the very same purpose that we use simulation for in our paper, only in the context of supply chains is presented in [18]. Paper [19] evaluates accuracy of kidney stone models generated from CT (computed tomography) medical images by comparing computer-generated models with 3D scanned ones using an approach similar to ours. The same problem of dimensional accuracy, only in the context of 3D printed camera casing models is discussed in [20]. A more general comparison of selected algorithms for the calculation of form deviation is presented in [21].

\section{PROBLEM STATEMENT AND METHODOLOGY}

For digital 3D reconstruction of a desired physical object, photogrammetry techniques require the user to capture a series of photos of the object under certain constraints. To achieve successful 3D reconstruction, these photos must have sufficient overlap, resolution, and sharpness [2]. A successful system requires the optimization of all parameters [2, 10]. Especially important is the number of cameras (it is possible to have more logical cameras than physical ones using, for instance, a mobile camera rig) which must be balanced against cost. Number of cameras refers to the number of different camera viewpoints from which photos have to be captured in the photogrammetric process. These photos can be taken by a number of physical camera devices equal to or less than the number of cameras. If conditions allow, all photos can be taken with a single physical camera device. In order to get a scanner which is both effective, cost-controlled, and produces repeatable output, the number of cameras and their position and orientation must be fixed.

Therefore, for the development of a photogrammetry-based 3D scanner, it is typically necessary to experiment with physical cameras, where different number of cameras, their viewpoints, and parameters are tested and tuned until the optimal configuration is discovered. Afterwards, the production version of the scanning system is developed based on the configuration which was empirically derived. This trial and error approach is, itself, slow and can result in delayed and inefficient scanning methodology. This means that there are several avenues for improvement in terms of cost, time, and quality in both the development and exploitation phase.

We instead propose to use a simulation approach to speed up the previously described process, lower the costs involved, and achieve the desired quality level of 3D reconstruction. The proposed approach is based on using virtual cameras and computer-generated photos from these cameras. All the time-consuming tests are now performed in a virtual, simulated environment. This considerably increases the speed and lowers the costs of 3D scanning system development.

General algorithm for the proposed simulation approach is shown in Fig. 1. It consists of the following steps:

1. Preparation of reference $3 D$ model/s;

2. Choosing the number of virtual cameras, based on the selected criteria;

3. Creation of a virtual 3D scene, cameras, and lighting;

4. Setup of a new virtual camera layout;

5. Setup/update of virtual cameras;

6. Placing reference $3 D$ model/s in virtual 3D scene;

7. Rendering of images from virtual cameras;

8. Embedding of EXIF data in images;

9. Processing images using photogrammetry-based reconstruction software (creation of 3D object reconstruction); 
10. Repeating steps 6-9 for all 3D models;

11. Evaluation of $3 \mathrm{D}$ object reconstruction results;

12. Repeating steps 5-11 until the desired quality for the given layout is achieved;

13. Save camera setup, camera layout, and camera number;

14. Repeating steps 4-12 until certain number of camera layouts for the given camera number are refined and evaluated;

15. Subjective 3D object reconstruction evaluation of different layouts;

16. Saving the camera setup, camera layout, and camera number;

17. Repeating steps 2-14 until certain combinations of camera numbers are refined and evaluated;

18. Subjective 3D object reconstruction evaluation of different camera numbers and layouts;

19. Selection of the best candidate for the production version of the photogrammetry-based 3D scanning system.

Target physical object/s and/or their classes are usually known in advance. This allows for using simulation to obtain an optimized layout beforehand. In order to simulate a physical target, a 3D model is used. Such a 3D model is called a reference 3D model since it is later used as a reference for comparison. Depending on the target, we distinguish two cases: a) target is a single physical object (e.g., Colosseum in Rome or Michelangelo's David); b) target is/are physical object class/es (e.g., human beings). To use simulation in the former case, at least one 3D model of the chosen physical object must be provided. Since simulation approach does not impose restrictions on the number of 3D models used for simulation, one or more or even all 3D models can be used. These models should preferably be high-detailed. When targets are object classes, a data set of 3D models should be carefully tailored, so that it is a statistically representative sample for each target object class. Afterwards, this data set is used in simulation. As in the previous case, high-detailed 3D models are preferred here too.

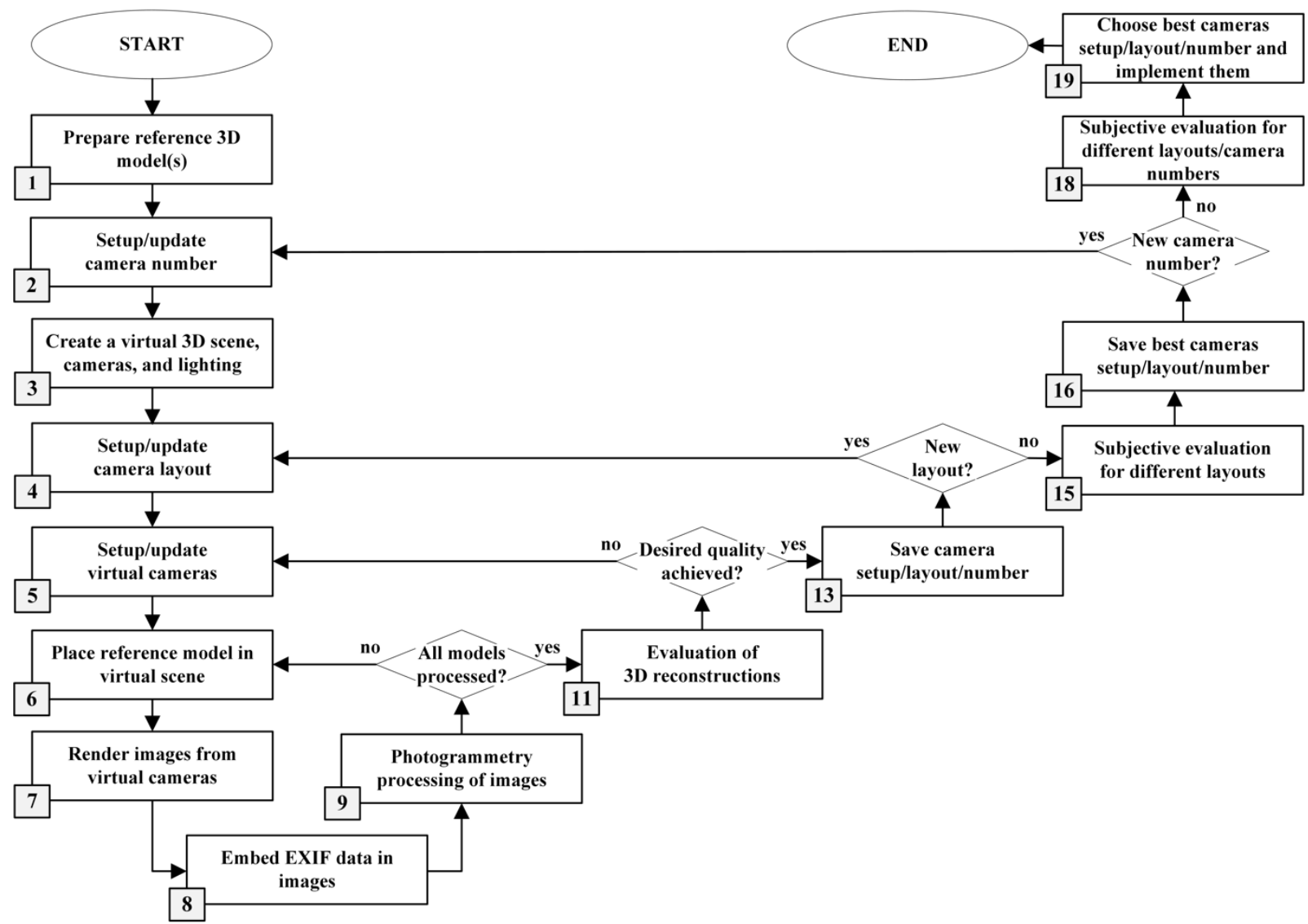

Figure 1: The algorithm for the proposed simulation approach. 
It is further necessary to define the number of virtual cameras used in simulation based on the selected criteria/strategy. One possible strategy would include starting with the maximum number of cameras economically possible and lowering that number in iterations, until minimal number is achieved. An interesting question here which remains for future research is how different strategies influence convergence and speed of finding the optimal solution.

Afterwards, a virtual 3D scene is created using the selected 3D modelling software (e.g., Blender, Autodesk 3D Studio Max, Autodesk Maya). Then, a number of virtual cameras are generated in this virtual 3D scene. In the proposed simulation approach, these virtual cameras are set to mimic real physical camera devices used for image acquisition.

Lighting in the simulated scene must be simulated, either by replicating the real-world conditions with as much fidelity as possible, or by assuming some simple model of lightning such as e.g. ambient lightning. Virtual camera layout and set camera parameters must be arranged either manually and/or automatically in the 3D modelling software. Automatic and semi-automatic support for camera simulation can be achieved using scripting language of the chosen 3D modelling software (e.g., MaxScript for 3D Studio Max).

The selected reference $3 D$ model from dataset is then placed in the centre of a virtual 3D scene, such that it is surrounded by virtual cameras. Images from virtual cameras are rendered using the 3D rendering software engine, either dedicated standalone software or built-in as 3D modelling software's renderer (e.g., Blender's Cycles).

Digital photographs from real cameras contain EXchangeable Image File format (EXIF) meta-data which describe photo capturing process and camera parameters. The EXIF data are used in many photogrammetry-based reconstruction software to improve processing. Thus, EXIF data from real photographs are embedded into images that simulate real camera photographs.

Rendered images with embedded EXIF data are then used as input for 2D-to-3D photogrammetric reconstruction performed using the selected software package (e.g., Agisoft PhotoScan). As a result of this process, a reconstruction of the reference $3 D$ model is created (henceforth $3 D$ reconstruction).

The rendered reference $3 D$ model is replaced with the next one from the data set and steps $6-9$ of the algorithm from Fig. 1 are repeated until $3 D$ reconstructions of all reference $3 D$ models have been obtained.

The quality of the $3 \mathrm{D}$ reconstruction is used to measure the quality of the simulated photogrammetry-based system. Simulated systems which generate best quality 3D reconstructions are most likely candidates to be developed into production versions of the photogrammetry-based 3D scanners. For this, it is necessary to develop or adopt an evaluation system which will be used uniformly over all simulations. As both trial-and-error and simulation approaches produce 3D reconstructions, the same evaluation system can be used both for manual and simulated real photogrammetry-based scanning system. Evaluation could include both objective and subjective components. Usually, objective evaluation will be the first one used to reject most of the worst-performing candidates (many of which are obviously bad). They can usually be applied automatically and are fast and impartial. In practice, issues with the 3D object reconstruction are common and inevitable. Thus, for finer decision making, subjective evaluation is necessary. Subjective evaluation can include visual inspection of the achieved 3D object reconstruction against the reference one. However, from the production point of view, it is not enough to conclude if and how much the 3D reconstruction differs from the reference. Photogrammetry-based 3D reconstruction process usually involves post-processing of the resulting reconstruction. Post-processing corrects a great number of issues of the $3 \mathrm{D}$ reconstruction process. Further, it increases its quality to the level which allows it to be practically used. The post-processing is usually done manually or semi-automatically by digital artists. Thus, 3D object reconstruction can be evaluated 
subjectively to estimate how hard and time-consuming it would be to amend insufficiencies and artefacts by manual work of a digital artist. An experienced digital artist can easily and in a relatively short time estimates how much time and effort is needed to post-process the 3D reconstruction. In the later phase of evaluation, it is possible to support subjective evaluations with objective evaluations which measure how many points and/or polygons 3D reconstruction contains in the head and/or body regions [14]. This is also a good indicator of how much time and effort will be needed in post-processing and it is usually used by digital artists in their estimations. As another topic for future research, it might be interesting to compare real-world camera reconstructions with simulated ones. Finally, according to the evaluation results, number of cameras, virtual camera layout, and virtual camera parameters are updated to better cover the reference $3 D$ model. The entire process is repeated until the desired precision and quality of reconstruction is achieved.

It should also be noticed that the proposed simulation approach is tool-agnostic and platform-agnostic, as it does not specify or require use of any specific 3D modelling or photogrammetric software and/or platform for its application.

\section{THE PROPOSED SOLUTION}

Based on the proposed simulation workflow, a simulator of a 3D scanning system for human avatar creation was developed (see Fig. 2). The target physical object class which is scanned is a human being standing in what's known as an A-pose (legs slightly apart and arms positioned at an approximately 30-degree angle away from the body).
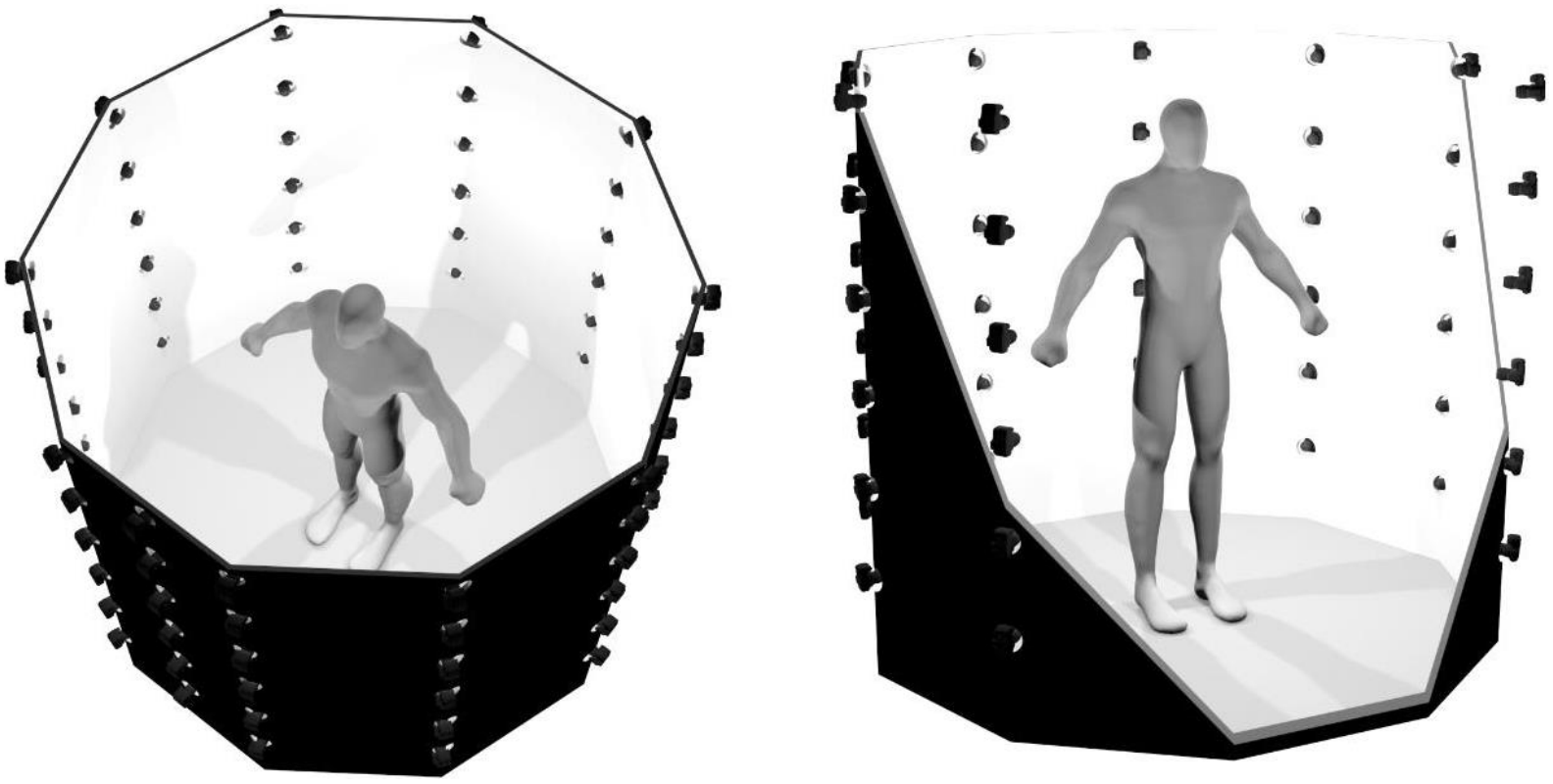

Figure 2: An image from the proposed simulator of a 3D scanning system for human avatar creation.

Since 2012, doob $A G$ has been scanning people using their proprietary photogrammetric scanners - dooblicators - and producing 3D digital avatars and human figurines using 3D printing technology. Doob $A G$ has a collection of more than 100,000 high-quality 3D models that have been post-processed by doob $A G$ digital artists. A statistically-representative set of high-quality human 3D models was created (reference 3D models), which are courtesy of doob $A G$ [22], as the gold standard for simulation process, henceforth originals dataset. This dataset has been carefully crafted to include human models of different ages, genders, heights, body types, and arrays of clothing. Thus, a broad range of real-world avatar scanning scenarios is covered. 
Autodesk 3D Studio Max 2018 [23] is used for creating the virtual 3D scene, camera layout development, lighting setup, and rendering of reference $3 D$ models based on virtual camera views. To achieve photorealistic rendering, 3D Studio Max's physical camera type is used to simulate a real-world digital camera. For each virtual camera, the following parameters are set manually: position, orientation, field of view, image resolution, and depth of field to match real-world camera parameters. For lighting sources, Omni light type is used as the best fit for the multiple omnidirectional flashes used in our system. Lighting calculation is the most computationally expensive step in rendering. Therefore, while more complex lightning setups are possible, they should be avoided wherever the integrity of the simulation does not demand it. In order to support automation of camera layout and lighting setup/update, several scripts are developed to load and save their parameters as JavaScript Object Notation (JSON) files.

The simulation uses the scanline renderer built into 3D Studio Max because it is simple, fast, and fit for purpose. Others [24] can be used instead in case of more complex setups. As images from real-world digital cameras have embedded EXIF data and these data are later used by photogrammetry-based software, rendered images are automatically provided with simulated EXIF data using ExifTool [25]. A script has been developed to automate embedding of EXIF data.

Rendered images are processed in order to obtain the $3 D$ reconstructions for each $3 D$ reference model using the Agisoft PhotoScan Professional photogrammetry-based reconstruction software [26]. The PhotoScan Professional is chosen for its high-quality 3D reconstruction and wide-spread use for photogrammetry-based 3D reconstruction, both in industry and research, especially in the field of cultural heritage [27]. In addition, since it is publicly available, it allows reproducible research. We use the built-in Python scripting support to automate PhotoScan processing and speed up the simulation process.

Furthermore, we developed a set of scripts to automate this process, as shown in Fig. 3. The main script listens for scan creation, i.e., images appearing on the predefined path. Afterwards, it creates a PhotoScan project file and adds images for processing. Image alignment step uses the SfM approach to extract both camera positions and orientations, as well as the sparse point cloud (sparse 3D reconstruction). The bounding box defines the part of the sparse point cloud which is of interest and that is used later for building the dense point cloud. Bounding box is set automatically to the maximum usable space in the simulator. Afterwards, dense point cloud is constructed, followed by creation of a textured mesh. When the 3D object reconstruction is complete, it is exported using standard 3D formats like OBJ or PLY. This procedure is repeated when the next scan arrives, until the user closes the application.

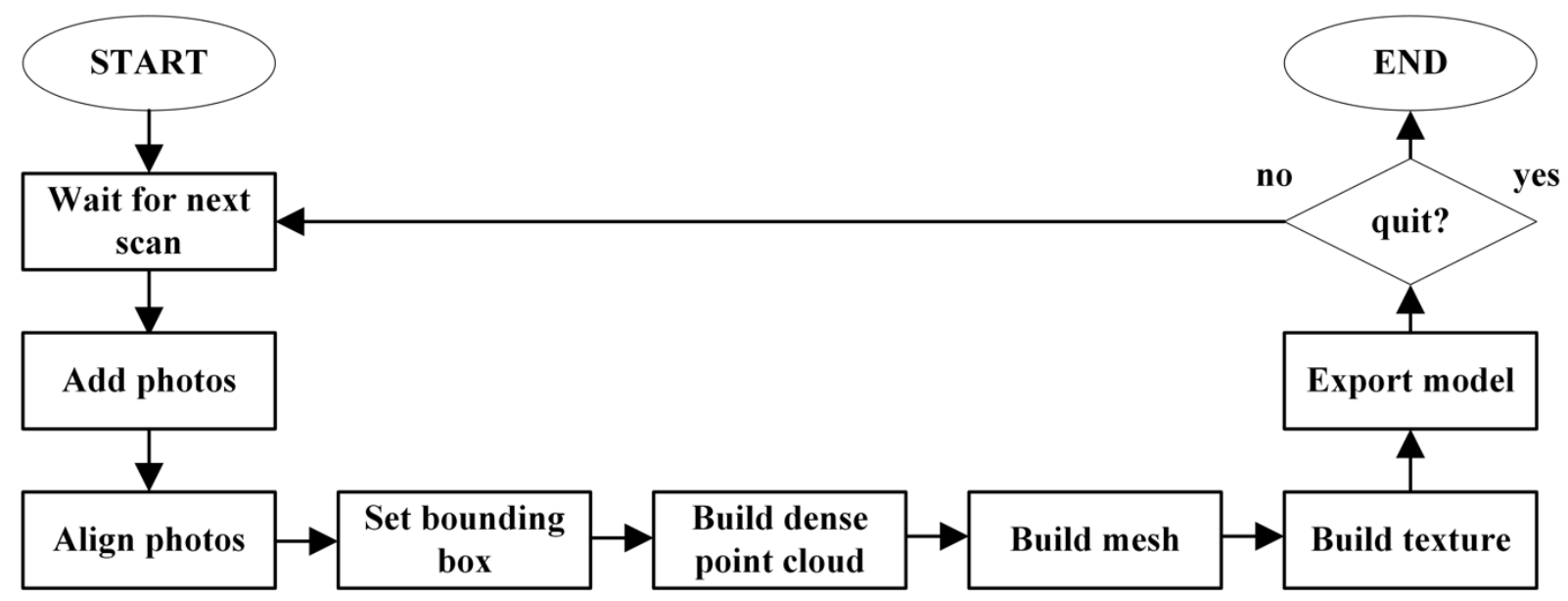

Figure 3: Automated PhotoScan processing workflow. 
To evaluate the $3 D$ reconstruction and - ultimately - the simulated photogrammetry-based system, we used both objective and subjective evaluation.

Objective evaluation is used to compare the $3 D$ reconstruction with the reference $3 D$ model. The CloudCompare open-source software [23] is used for comparison of the $3 D$ reconstruction with its reference $3 D$ model. For each 3D model pair, models are first aligned and finely registered. Then the distance measure is obtained using Cloud-to-Mesh Distance [28]. For visualization of distance measure, height map is used, where different distances are mapped to different colours. By default, Blue-Green-Yellow-Red colour scale is used, meaning that smallest computed distances will be coloured in blue, larger in green and yellow, whereas the greatest distances will be coloured in red. Visualization of deviation of the $3 D$ reconstruction from the reference model is used to deduce larger deviations in the $3 D$ reconstruction, such as bad coverage of certain parts of the model, whereas distance measure is used for more precise comparison when good coverage has already been achieved (e.g., see Fig. 6). As the simulation goal was to minimize these distances for all models in the dataset, a weighted sum of distances is used for calculation of how good certain simulation iteration is. A weighted sum of distances is employed as not all reference models are of equal importance (e.g. according to height statistics, not all human heights are equally represented in global population).

Subjective evaluation is used in two ways. The first use is during the refinement of virtual camera parameters (the $11^{\text {th }}$ step in the simulation algorithm). After distance evaluation and visual inspection of the height map steps, subjective evaluation of the $3 D$ reconstruction is performed by a three-member panel of digital artists. Based on their expertise, $3 D$ reconstruction is evaluated from the perspective of how much post-processing is required to make it usable for production. According to evaluation results, camera layout is manually adjusted in $3 D$ Studio Max and the procedure is repeated until the target distance level and quality is achieved. The second use of subjective evaluation is to choose between different layouts with either the layout or the number of virtual cameras being varied. The same threemember panel of digital artists choses, based on their experience and estimation of how much effort is needed for post-processing, the most likely candidate for a photogrammetry-based system. In each iteration, processing time is measured and summarized in order to compute the full time required for the entire simulation process.

\section{EXPERIMENTS}

In order to validate the proposed approach, we devised and conducted the following experiments.

First, to test whether our simulator successfully emulates the behaviour of the real-world system, we compared it to the physical 3D scanning system called dooblicator 1.0, courtesy of doob AG [13]. Dooblicator 1.0 is a room-size scanning booth containing 54 DSLR (Digital Single-Lens Reflex) cameras arranged in nine columns with six cameras per column (see Fig. 4). We used the originals dataset (see Section 4 for more details) for performing this experiment. Lighting, virtual camera layout and virtual camera parameters were carefully set to match the parameters of the dooblicator 1.0 setup. For each of the 3D models from originals dataset, we ran a simulation which yielded its $3 \mathrm{D}$ reconstruction.

Afterwards, 3D models from the originals dataset and their respective $3 D$ reconstructions were first compared using the CloudCompare open-source software. Mean cloud to mesh distance calculated is 0.0005173 , with the standard deviation of 0.001056 ; a boxplot of this dataset is shown in Fig. 5. Later, $3 D$ reconstructions were evaluated by doob AG digital artists (forming an expert panel consisting of three members who processed thousands of scans). Experts did not find any significant visual deficiencies. As there were no significant 
deficiencies, both by visual inspection (subjective evaluation) or statistically significant deviations between the originals and the respective $3 D$ reconstructions (objective evaluation), we can conclude that the simulation approach is valid. The same experimental protocol can be used to iteratively tweak some parameter of the setup and, thus, effect refinements in the design.

a)

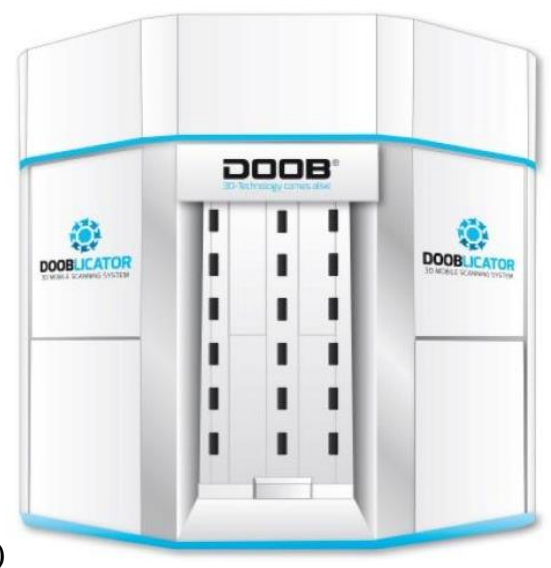

b)

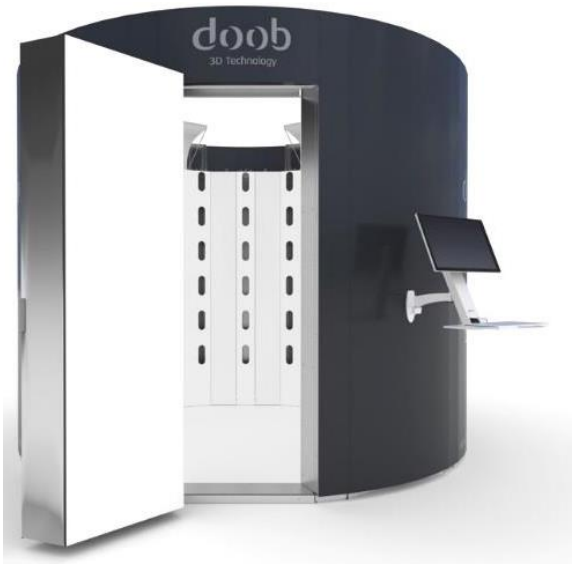

Figure 4: dooblicator 3D scanning system for creation of human avatars (courtesy of doob $A G$ ): a) version 1.0 ; b) version 2.0 .

Cloud to Mesh Distance

$\square$ mean $\square$ standard deviation

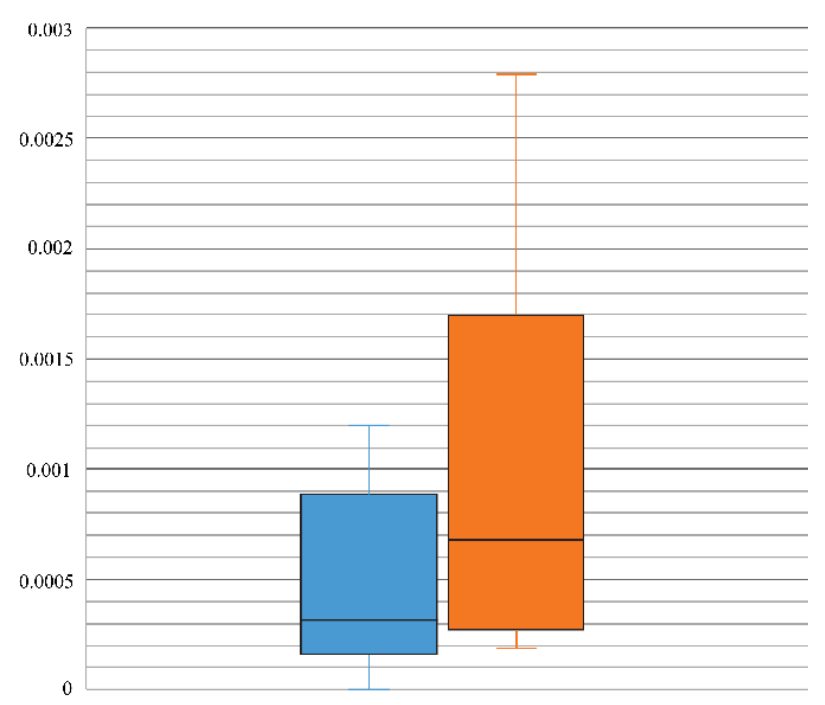

Figure 5: Boxplot of Cloud-to-Mesh Distance.

The second experiment was aimed at testing whether our simulator truly shortens time and reduces costs for the development of 3D scanning systems using photogrammetry-based data acquisition. Doob $A G$ was planning to develop a new version of the dooblicator - the dooblicator 2.0. So, we used our simulator to create a prototype proposal (the experimental group), while doob $A G$ was developing a prototype using the trial-and-error approach with real hardware (the control group). The camera manufacturer and model, the number of cameras, and the dimensions of the scanner were set in advance as constraints, both for practical reasons and to make sure the approaches were comparable. We also restricted the duration of the experiment to three months. Duration of the experiment was restricted in order to fit the real-world premium on quick turnaround and efficient business processes and was supported by $\operatorname{doob} A G$ with its experience in building scanning systems. 
After the period of three months had expired, the experimental group results were compared with the control group results. During the experiments, 47 volunteers were scanned in both scanner prototypes and 3D reconstructions were processed using the same pipeline. For each volunteer and for each prototype, reference $3 D$ models were made to the highest standards of quality by doob $A G 3 \mathrm{D}$ artists. Both the experimental and the control group's 3D reconstructions were evaluated objectively using CloudCompare as in the first experiment. CloudCompare showed a clear advantage of the simulation-based approach, as distances and completeness of 3D reconstructions were better than in the control group. Mean distances and standard deviations for experimental and control group are shown in Table I. Distances and deviations of control group were higher by order of magnitude (16.62 and 8.03 times, respectively, than in the experimental group).

Table I: Mean distances and standard deviations for 3D reconstructions obtained in the control and in the experimental groups.

\begin{tabular}{|l|c|c|}
\cline { 2 - 3 } \multicolumn{1}{c|}{} & Mean distance & Standard deviation \\
\hline Control group & 0.408828 & 0.474662 \\
\hline Experimental group & 0.024601 & 0.059086 \\
\hline
\end{tabular}

These large differences are caused by the completeness issues of $3 D$ reconstructions and the lack of fine details on scans taken in the control group. Example of hand completeness issues of a reconstruction from a volunteer's scan from the control group, visualized using default height mapping colour scale, is shown on the left side of Fig. 6. On the right side of Fig. 6, the reconstruction of the same volunteer's hands from the experimental group is shown. From Fig. 6, one could observe that hands are completely reconstructed in the experimental group scan and that distances measured are small (as they are presented in blue). In contrast, there are significant deficiencies in the control group scan; parts of hands are either not reconstructed at all and very large distances from the ground truth are measured marked with yellow and orange). These results are direct consequence of the trial-and-error approach used in the control group. Namely, it is hard to position and orient tens of cameras correctly manually to capture all of the relevant details with sufficient overlap. Subjective evaluation by doob $A G$ experts confirmed conclusions of the objective evaluation - the experimental group scan $3 D$ reconstructions were better, both in terms of completeness and level of details reconstructed.
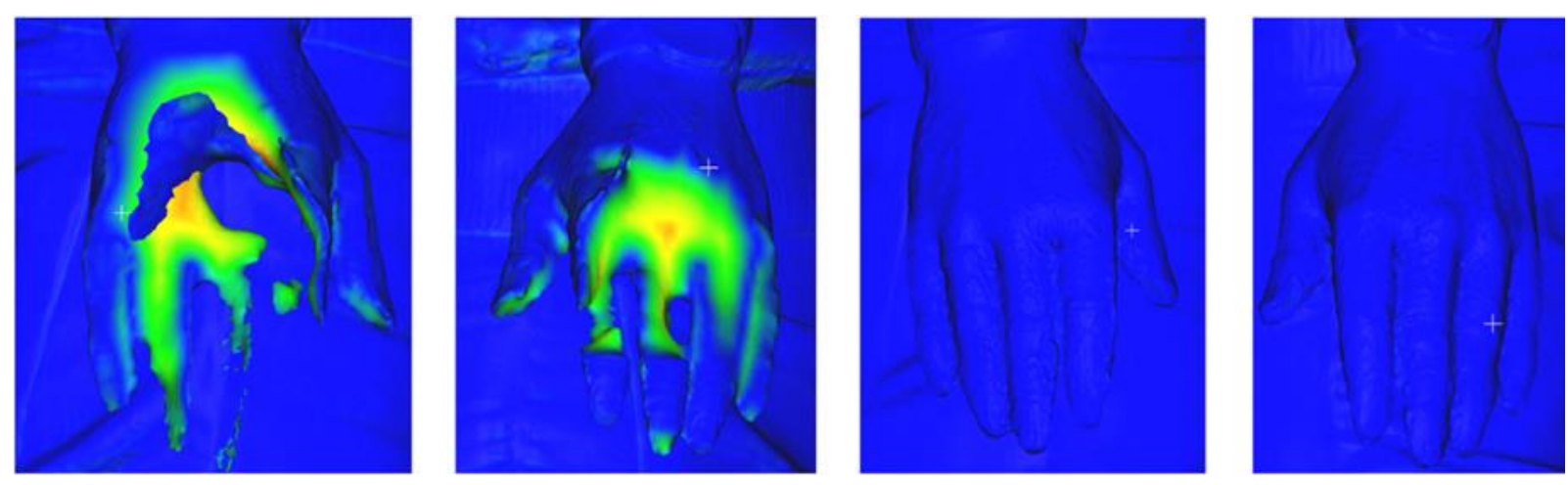

Figure 6: Hands completeness comparison for the left and the right hand, obtained from the control group scan, are shown as first two images from the left; the remaining two images show the reconstructions of the same objects using the experimental group scan obtained with the proposed simulator.

Evaluation showed that the scanner prototype, developed according to the simulationbased approach, offers higher-quality of $3 D$ reconstructions than the one used in control 
group. In addition, the experimental group, due to the fast and inexpensive simulation approach, came up with several camera layout candidates in just one week. Candidates were evaluated, as described in Section 4, and the best candidate was chosen. The scanner prototype was built only once, according to the best candidate simulation proposal. In contrast, the control group used all three months trying to get good quality and built and tear down several prototypes before reaching satisfactory results.

According to the results obtained in this experiment, doob $A G$ decided to use our prototype as the basis for dooblicator 2.0. During the period of one year, several thousands of customers were successfully scanned in the A-pose using the settings of the simulated approach which validated the proposed approach in production use.

\section{CONCLUSION}

In this paper, we presented a novel simulator of a 3D scanning system using photogrammetrybased 3D data acquisition. To the best of our knowledge, no similar simulators, aimed at reducing cost and improving performance of 3D scanning based on close-range photogrammetry, have been proposed in the literature.

The proposed approach is characterized by a platform-agnostic simulation workflow. We put the value of the discussed simulator to the test using as an experiment the process of developing a 3D scanning system for human avatar creation. While the simulations performed do not take into account every camera parameter, offering no provision for simulating complex lighting conditions, lens distortions, and sensor noise, the results of their use in practice show that what they do simulate is sufficient to improve production efficiency and quality and thus solve some of the existing problems in 3D scanning system design, development, and production and improve the outcomes of the 3D scanning process.

In order to validate the proposed approach, we devised and conducted two separate sets of experiments focused on 3D human body scanning for avatar creation. The results of the experiments confirm that the application of the proposed approach leads to an improved quality of 3D object reconstruction, as well as lowers the cost and shortens the time required for the construction of 3D scanning systems used for human body scanning.

As future work, we plan to automate the camera layout discovery according to a small set of initial input parameters from the user. Another improvement of the developed simulator would be full automation of the process of simulation and verification. Finally, we plan to extend our simulation approach to other 3D scanning techniques.

\section{ACKNOWLEDGEMENTS}

The authors would like to thank Doob Group AG for the support and dataset provided for this research. The reported research is also partly supported by the Ministry of Education, Science, and Technological Development of the Republic of Serbia, projects TR32044 (2011-2019), ON174026 (2011-2019), and III44006 (2011-2019).

\section{REFERENCES}

[1] Zhao, F.; Loy, S. C. (2017). A practical approach to ascertaining the accuracy and resolution of post rendered 3D models acquired originally via 3D digitization, Proceedings of the $7^{\text {th }}$ International Conference on Digital Presentation and Preservation of Cultural and Scientific Heritage, Vol. 7, 89-105

[2] Hartley, R.; Zisserman, A. (2004). Multiple View Geometry in Computer Vision, $2^{\text {nd }}$ edition, Cambridge University Press, Cambridge

[3] Piatti, E. J.; Lerma, L. J. (2013). Virtual worlds for photogrammetric image-based simulation and learning, The Photogrammetric Record, Vol. 28, No. 141, 27-42, doi:10.1111/phor.12001 
[4] Gartner IT Glossary. Digitalization, from https://www.gartner.com/it-glossary/digitalization/, accessed on 25-08-2018

[5] Joglekar, J.; Gedam, S. S. (2011). Area based stereo image matching technique using Hausdorff distance and texture analysis, International Archives of the Photogrammetry, Remote Sensing and Spatial Information Sciences, Vol. XXXVIII-3/W22, 109-114

[6] Berdic, N.; Dragan, D.; Mihic, S.; Anisic, Z. (2017). Creation and usage of 3D full body avatars, Annals of Faculty Engineering Hunedoara - International Journal of Engineering, Vol. 15, No. 1, 29-34

[7] Dragan, D.; Anišić, Z.; Mihić, S.; Puhalac, V. (2018). 3D Avatar Platforms: Tomorrow's Gateways for Digitized Persons into Virtual Worlds, Hankammer, S.; Nielsen, K.; Piller, F.; Schuh, G.; Wang, N. (Eds.), Customization 4.0, Springer Proceedings in Business and Economics, Springer, Cham, 141-155, doi:10.1007/978-3-319-77556-2 9

[8] Schönberger, J. L.; Frahm, J.-M. (2016). Structure-from-motion revisited, Proceedings of the $29^{\text {th }}$ IEEE Conference on Computer Vision and Pattern Recognition (CVPR), 4104-4113

[9] Lowe, D. G. (2004). Distinctive image features from scale-invariant keypoints, International Journal of Computer Vision, Vol. 60, No. 2, 91-110, doi:10.1023/B:VISI.0000029664.99615.94

[10] Luhmann, T.; Robson, S.; Kyle, S.; Boehm, J. (2013). Close-Range Photogrammetry and 3D Imaging, $2^{\text {nd }}$ edition, De Gruyter, Berlin

[11] Arbutina, M.; Dragan, D.; Mihić, S.; Anišić, Z. (2017). Review of 3D body scanning systems, Acta Technica Corviniensis - Bulletin of Engineering, Vol. 10, No. 1, 17-24

[12] Harari, Y.; Bechar, A.; Raschke, U.; Riemer, R. (2017). Automated simulation-based workplace design that considers ergonomics and productivity, International Journal of Simulation Modelling, Vol. 16, No. 1, 5-18, doi:10.2507/IJSIMM16(1)1.355

[13] Doob Group AG (2018). Dooblicator - The World's First Mobile 3D-Scanning System, from http://www.doobgroup.com/en/erleben/dooblicator/, accessed on 15-08-2018

[14] Dragan, D.; Mihic, S.; Anisic, Z.; Lukovic, I. (2015). Role of background subtraction in creating human body point clouds from photographs, Proceedings of the $6^{\text {th }}$ International Conference on $3 D$ Body Scanning Technologies, 210-217

[15] Khan, M. A. A.; Sheikh, A. K. (2018). A comparative study of simulation software for modelling metal casting processes, International Journal of Simulation Modelling, Vol. 17, No. 2, 197-209, doi:10.2507/IJSIMM17(2)402

[16] Yang, L.; Zheng, M. L. (2017). Simulation and analysis of ball-end milling of panel moulds based on Deform 3D, International Journal of Simulation Modelling, Vol. 16, No. 2, 343-356, doi:10.2507/IJSIMM16(2)CO9

[17] Vickovic, L.; Gotovac, S.; Celar, S. (2016). Simulation-based performance analysis of the ALICE mass storage system, International Journal of Simulation Modelling, Vol. 15, No. 1, 7082, doi:10.2507/IJSIMM15(1)6.325

[18] Ramírez, C. S.; López, D. R. M.; Hernández, G. A.; Alcaraz, J. L. G.; Mendoza, D. A. T. (2016). Simulation software as a tool for supply chain analysis and improvement, Computer Science and Information Systems, Vol. 13, No. 3, 983-998, doi:10.2298/CSIS160803039S

[19] Galeta, T.; Pakši, I.; Šišić, D.; Knežević, M. (2017). Comparison of 3D scanned kidney stone model versus computer-generated models from medical images, Advances in Production Engineering \& Management, Vol. 12, No. 3, 254-264, doi:10.14743/apem2017.3.256

[20] Mandić, M.; Galeta, T.; Raos, P.; Jugović, V. (2016). Dimensional accuracy of camera casing models 3D printed on Mcor IRIS: A case study, Advances in Production Engineering \& Management, Vol. 11, No. 4, 324-332, doi:10.14743/apem2016.4.230

[21] Magdziak, M. (2018). A comparison of selected algorithms of form deviation calculation, Technical Gazette, Vol. 25, No. 5, 1389-1394, doi:10.17559/TV-20170609103530

[22] Doob Group AG. doob 3D Technology, from http://www.doobgroup.com/en/discover/discover/, accessed on 05-08-2018

[23] Autodesk, Inc. Autodesk 3D Studio Max, from https://www.autodesk.com/products/3dsmax/overview, accessed on 07-08-2018

[24] SolidAngle. Arnold global illumination renderer, from https://www.solidangle.com/arnold/, accessed 27-07-2018 
[25] Harvey, P. ExifTool by Phil Harvey - Read, Write, and Edit Meta Information!, from https://www.sno.phy.queensu.ca/ phil/exiftool/, accessed on 07-03-2018

[26] Agisoft, LLC. Agisoft PhotoScan, from http://www.agisoft.com/features/professional-edition/, accessed on 07-03-2018

[27] De Reu, J.; Plets, G.; Verhoeven, G.; De Smedt, P.; Bats, M.; Cherrette, B.; De Maeyer, W.; Deconynck, D.; Herremans, D.; Laloo, P.; Van Meirvenne, M.; De Clercq, W. (2013). Towards a three-dimensional cost-effective registration of the archaeological heritage, Journal of Archaeological Science, Vol. 40, No. 2, 1108-1121, doi:10.1016/j.jas.2012.08.040

[28] CloudCompare. CloudCompare - 3D point cloud and mesh processing software, from http://www.danielgm.net/cc/, accessed on 27-08-2018 